\title{
Certificação ISO 9001:2000 - a Experiência da SoftExport
}

\author{
Raimundo Sales Neto e Azevedo ${ }^{1,2}$, Ana Cristina M.Matos ${ }^{2}$, Marum Simão Filho ${ }^{1}$, \\ Flávio Lenz César ${ }^{1}$, Arnaldo Dias Belchior ${ }^{2}$ \\ sales.mia@unifor.br, anacristina.m@uol.com.br,marum@softexport.com.br, \\ lenz@,softexport.com.br, belchior@unifor.br \\ ${ }^{(1)}$ SoftExport \\ ${ }^{(2)}$ Universidade de Fortaleza (Mestrado em Informática Aplicada) \\ Av. Washington Soares, 1321, 60.811-341, Fortaleza-Ceará
}

\begin{abstract}
Resumo
Este trabalho relata a experiência do processo de certificação ISO 9001:2000 da fábrica de software, SoftExport. A implantação do Sistema de Gestão da Qualidade (SGQ) na fábrica passou a orientar os processos fundamentais de desenvolvimento de software, os processos organizacionais e os processos de apoio, estruturando todas as rotinas da empresa e ampliando a visão da mesma em relação a seus próprios produtos, processos, colaboradores e da relação com seus clientes. O processo de certificação foi conduzido através de um conjunto de atividades estruturadas a partir da ISO/IEC 12207, diferenciando-se da seqüência lógica de implantação dos itens da ISO 9001. Isto deu uma maior agilidade no processo de implantação do SGQ na fábrica de software.

Palavras-chave: processo de software, fábrica de software, ISO 9001, ISO/IEC 12207.
\end{abstract}

\begin{abstract}
This work relates the experience in the certification ISO 9001:2000 process of the software factory SoftExport. The Quality Management System (QMS) implementation in the factory guided the fundamental processes of the software development, the organizational processes and the support processes, structuring all the practices in the company and extending its vision in relation to their own products, processes, collaborators and clients. The certification process was conducted through a set of structured activities based upon ISO/IEC 12207, in contrast to the logical sequence of implementation items in ISO 9001. As a result, this work provided greater agility in the implementation process of the QMS in the software factory.
\end{abstract}

Keywords: software process, ISO certification

\section{Introdução}

A certificação ISO 9000 é reconhecida em todo o mundo em vários setores produtivos. A conquista da certificação ISO leva a organização a um nível de padrão internacional de qualidade em seus processos. No entanto, no âmbito de um determinado setor, não é possível diferenciar o nível de maturidade de uma empresa em relação a outra, em um conjunto de empresas que tenham recebido a certificação ISO 9000, a não ser pelo escopo de certificação, pela qualidade do certificador e pelo tempo pelo qual a certificação vem sendo mantida $[5,6]$.

A ISO 9000 é um padrão internacional de qualidade, que aplica o gerenciamento da qualidade do processo para gerar produtos, que atentam às expectativas de seus usuários. Esses padrões foram criados sob a premissa de que, se o desenvolvimento e o gerenciamento do sistema são de boa qualidade, então, o produto ou o serviço resultante também será de boa qualidade. Um sistema de Gestão da qualidade em conformidade com a ISO 9000 assegurará que seu processo de desenvolvimento possui um nível de controle, disciplina e repetibilidade, garantindo a qualidade de seus produtos. Neste contexto, a SoftExport, uma fábrica de software, que utiliza a tecnologia J2EE, buscou a certificação ISO 9001:2000 (ISO 9001, 2000). A empresa, com seus 21 colaboradores (pequena empresa, segundo o do MCT/SEPIN), é composta de técnicos e tem a natural dificuldade nas suas rotinas administrativas.

Este trabalho está organizado da seguinte forma: a seção 2 descreve brevemente as normas ISO 9001:2000 [3] e ISO/IEC [4]; a seção 3 apresenta a fábrica de software 
SoftExport; a seção 4 discorre sobre a experiência de certificação na fábrica; a seção 5 mostra as lições aprendidas; e a seção 6 exibe as principais conclusões deste trabalho.

\section{A ISO 9001 ISO/IEC 12207}

A ISO 9000 [2], e ISO 9001 [3] foram elaboradas para orientar as organizações na implementação eficaz de seu sistema de gestão da qualidade. A partir da implementação desse sistema, cujo foco é a garantia da qualidade, a empresa estará preparada para tratar com maior agilidade e eficácia a ocorrência de problemas. A qualidade vai sendo complementada em seus processos e produtos, em uma ação de melhoria contínua.

O sistema de gestão da qualidade de uma empresa possui um conjunto de diretrizes, que permite a seus clientes avaliar a capacidade dessa organização em fornecer produtos e serviços, que atendam os requisitos especificados de forma consistente, fornecendo ainda uma estrutura para melhoria contínua do desempenho da organização. Uma empresa, que deseja certificar seu sistema de gestão da qualidade segundo a ISO 9001, deverá considerar as seguintes questões:

- conhecer e demonstrar sua capacidade em atender os requisitos dos clientes;

- planejar e documentar todas as atividade que afetam a qualidade;

- qualificar pessoas nas competências necessárias à realização de tarefas;

- identificar e disponibilizar recursos materiais e humanos necessários para manter o sistema da qualidade;

- registrar a execução das atividades;

- prevenir as não-conformidades e, se ocorrerem, devem ser registradas e tratadas;

- identificar os processos críticos para a satisfação dos clientes;

- manter um programa contínuo de avaliação do desempenho do sistema.

A organização deve aplicar sua política da qualidade para evidenciar o comprometimento da alta direção para com a qualidade. Deve estar adequada aos propósitos da organização, ser verdadeira e refletir os valores da empresa para todos os clientes, funcionários e demais interessados.

A norma recomenda que, para um gerenciamento eficaz, a melhor forma de atender aos requisitos é a criação de procedimentos documentados. Os procedimentos são uma forma especificada para desenvolver uma atividade. Ela exige apenas a elaboração de seis procedimentos: Controle de documentos, Controle de registros, Auditoria interna, Controle da não conformidade de produtos, Ação corretiva, e Ação preventiva.

A existência de procedimentos, instruções e registros de trabalho formalizam todas as atividades que afetam a qualidade. Isto exige a participação de todos os indivíduos da organização. Portanto, a conscientização para com a qualidade aumenta, uma vez que todos participam diretamente da implementação do sistema da qualidade, pois são os principais responsáveis pelas atividades da empresa.

A ISO/IEC 12207 [4] descreve o ciclo de vida de software em três macro-processos: processos fundamentais, processos de apoio, e processos organizacionais. Os processos fundamentais agrupam as partes que integram diretamente a produção do software, sendo eles: aquisição, fornecimento, desenvolvimento, operação, e manutenção. Os processos de apoio auxiliam outros processos na busca do sucesso e da qualidade do projeto e são formados por: documentação, gerência de configuração, garantia da qualidade, verificação, validação, revisão conjunta, auditoria, e resolução de problema. Os processos organizacionais envolvem tipicamente políticas e práticas institucionais, sendo compostos por: gerência, infraestrutura, treinamento, e melhoria. Cada processo e composto de um conjunto de atividades, que por sua vez é composta de um conjunto de tarefas. 


\section{A SoftExport}

A SoftExport é uma fábrica de software localizada em Fortaleza e atua também nas regiões Sul e Sudeste do País, que tem como principal objetivo a prestação de serviços de desenvolvimento de software, fazendo uso da tecnologia J2EE. A empresa nasceu com perspectivas de exportação de software e há um planejamento para exportação de software para mercados internacionais a ser implementado, com a abertura de frentes comerciais em países da Europa e nos Estados Unidos.

Dentro do plano de negócios inicial, sabia-se que, para atingir níveis de confiabilidade, produtividade e qualidade, a empresa deveria passar pelo amadurecimento de seus processos internos, que englobam, desde metodologias de desenvolvimento, até programas de qualificação de capital humano.

Uma fábrica de software tem como "linha de produção" a execução de projetos de desenvolvimento de software. Garantir que esses projetos sejam executados dentro de padrões de qualidade, prazo e custo é muito mais uma questão de sobrevivência, do que uma questão puramente comercial. Além disso, todos os outros processos auxiliares que influenciam diretamente nessa "linha de produção", como formação e capacitação de capital humano, aquisições, atualização tecnológica, etc. devem também estar garantidos por padrões de qualidade. Também não pode conviver com atrasos de projetos, falta de estimativas reais de custos, e com a qualidade dos produtos quase sempre aquém do esperado.

Visando atingir níveis aceitáveis de produtividade e qualidade, a SoftExport constituiu um Grupo da qualidade tendo entre seus colaboradores vários profissionais com mestrado em computação e com certificados pela SUN, Microsoft, e PMP (Project Management Professional).

\section{A Experiência de Certificação}

O Sistema de Gestão da Qualidade (SGQ) da SoftExport foi discutido e adotado pela alta administração da empresa, visando centralizar as ações de desenvolvimento de software referentes à qualidade de processos e de produtos, como também ao atendimento das necessidades de seus clientes.

O início da implantação do SGQ aconteceu abril de 2003, e a auditoria de certificação ISO 9001 estará ocorrendo em 25 e 26 de março de 2004. A pré-auditoria oficial ocorrida em fevereiro de 2004, apontou 5 observações e 12 sugestões de melhoria, ressaltando as facilidade dos ajustes e validando o SGQ.

A implantação do SGQ na SoftExport foi realizada a partir dos 3 macro-processos da ISO/IEC 12207 [4], que servem como tripé para as atividades da fábrica de software. Esta estrutura atende aos requisitos da ISO 9001 e reflete uma visão sistêmica da empresa e de suas rotinas, tanto as rotinas de produção, quanto as de administração. Com isto, as atividades e terefas descritas no dia-a-dia da empresa de software, levaram seus colaboradores a identificarem e situarem suas responsabilidades nos seus vários processos formalizados. $\mathrm{O}$ mapeamento da ISO 9001 com a ISO/IEC 12207 apoia-se principalmente nesta identificação, possibilitando também à ISO 9001 uma adequação ao perfil técnico de uma fãbrica de software. Desta forma, todos os requisitos para a certificação ISO 9001 foram enquadrados nos processos de ciclo de vida do software, além de outros procedimentos importantes e necessários que foram gerados.

O Planejamento da Qualidade é realizado a partir das diretrizes determinadas pela Direção da SoftExport na Política da Qualidade e é coerente com os Objetivos para Qualidade. Nesse planejamento, são levados em consideração os requisitos de gestão da qualidade e a implementação de melhorias contínuas necessárias para atender à Política da 
Qualidade e manter a integridade do SGQ. Essas melhorias são operacionalizadas mediante a implementação de mudanças no SGQ, de forma a mantê-lo permanentemente atualizado e em consonância com os requisitos especificados pelos clientes. As atividades que influenciam direta ou indiretamente a qualidade, são realizadas de forma estruturada. Essa estrutura inclui atividades de verificação e de controle da qualidade nas diversas etapas dos processos, as quais são realizadas de forma planejada e obedecendo a requisitos e instruções definidas nos procedimentos documentados do SGQ.

O SGQ é mantido atualizado e sua eficácia é melhorada de forma contínua, de acordo com o estabelecido na sua Política da Qualidade e pelas diretrizes básicas da superior administração. Suas características estão descritas em procedimentos documentados, os quais são elaborados de forma a descrever a metodologia de realização das atividades relacionadas com a qualidade de processos e produtos. A estrutura documental do SGQ é composta por:

- Leis, decretos, portarias (Documentos legais)

- Manual da Qualidade (Documento Principal do SGQ)

- Procedimentos (Documentos Normalizadores)

- Tutorais (Documentos Operacionais)

- Formulários/Modelos (Suporte para registros de dados)

- Registros (Resultados / evidências)

O Manual da Qualidade inclui: a descrição do escopo de certificação, as políticas da qualidade, faz referência aos procedimentos documentados usados nas diferentes atividades relacionadas com os processos administrativos e produtivos, e descreve a interação entre os processos do SGQ.

As atividades que influenciam direta ou indiretamente a qualidade dos processos ou produtos, são descritas nos Procedimentos e Tutoriais, que são utilizados para assegurar a correta realização das mesmas e o atendimento aos requisitos especificados. No SGQ da fábrica de software, existem 13 procedimentos documentados:

- Procedimentos organizacionais: contribuem para a melhoria da fábrica e estão fora do domínio de um único projeto. São eles:

- SQ.PO21: Procedimento para segurança;

- SQ.PO23: Procedimento de responsabilidades da direção;

- SQ.PO24: Procedimento para qualificação de fornecedores;

- SQ.PO27: Procedimento de qualificação profissional;

- SQ.PO30: Programa Estratégico de Qualificação.

- Procedimentos de apoio: auxiliam e contribuem para o sucesso e a qualidade do projeto/produto.

- SQ.PA01: Procedimento de controle de documentos;

- SQ.PA02: Procedimento de controle de registros do sistema da qualidade;

- SQ.PA03: Procedimentos para auditorias internas da qualidade;

- SQ.PA04: Procedimento de controle de produto não conforme;

- SQ.PA05: Procedimento para ação corretiva e ação preventiva;

- SQ.PA28: Procedimento para descrever o SGQ.

- Procedimentos Fundamentais: usados diretamente no ciclo de vida do software.

- SQ.PF20: Procedimento de projeto e desenvolvimento de sistemas;

- MUSSE: Metodologia Unificada de Desenvolvimento de Software da fábrica.

Os procedimentos determinam (ou fazem referência), quando necessário, à utilização de documentos operacionais, técnicos e/ou para suporte de registros e dados, tais como tutorais e formulários. Os Registros da qualidade necessários são definidos nas diferentes fases operacionais, assim como os formulários aplicáveis como suporte desta informação e dos dados. A responsabilidade pela gestão dos processos descritos nos procedimentos 
documentados está definida no próprio corpo de cada documento, exceto no caso de formulários, onde não é requerido. Quando necessária, a implementação dos documentos do SGQ é precedida de treinamento das funções envolvidas, de forma a garantir a correta interpretação e aplicação dos requisitos especificados.

\subsection{Roteiro das Atividades}

Os trabalhos para implantação do SGQ e a posterior certificação da empresa foram divididos em atividades desenvolvidas pelos participantes, e estão relacionadas na Tabela 1. As atividades dessa tabela estão ordenadas de forma a representar a seqüência no processo de certificação ISO 9001:2000 da SoftExport. Essas atividades, para uma melhor compreensão, ainda são caracterizadas no seguinte modo:

- Local: corresponde à seção da ISO 9001 onde a atividade está localizada. A atividade não prevista na ISO, mas considerada necessária, é marcada com " $N$ ".

- Modo: atividades seqüenciais $(S)$ ou desenvolvidas de forma paralela $(P)$.

- Tipo: atividade executada uma única vez $(U)$, ao longo do processo de certificação de forma contínua $(C)$, executada de forma secionada $(D)$, e repetidas vezes $(R)$.

\section{Tabela 1: Atividades do projeto SGQ}

\begin{tabular}{|l|c|c|c|}
\hline ATIVIDADES & Local & Modo & Tipo \\
\hline Abertura dos trabalhos, apresentação do projeto SGQ. & $N$ & $S$ & $U$ \\
\hline Levantamento dos processos e produtos da empresa. & 4 & $P$ & $D$ \\
\hline Estudo dos processos de desenvolvimento da empresa. & 4 & $P$ & $D$ \\
\hline Estudo dos produtos de software oferecidos pela empresa. & 4 & $P$ & $D$ \\
\hline Estudo da norma ISO 9000:2000. & $N$ & $P$ & $D$ \\
\hline Definição da política de qualidade da empresa: Missão, Visão do Futuro, Valores e Princípios. & 4 & $S$ & $U$ \\
\hline $\begin{array}{l}\text { Objetivos para a qualidade: Definir objetivos, Estabelecer metas, Estabelecer prazos, Estabelecer } \\
\text { responsáveis, Estabelecer padrões e indicadores de desempenho. }\end{array}$ & 4 & $S$ & $U$ \\
\hline $\begin{array}{l}\text { Elaborar procedimentos documentados obrigatórios requeridos pela norma: Controle de documentos, } \\
\text { Controle de registros, Controle de produtos em não-conformidade, Controle de ações corretivas, } \\
\text { Controle de ações preventivas, Controle de auditorias internas. }\end{array}$ & & & $S$ \\
\hline $\begin{array}{l}\text { Elaborar procedimentos documentados não obrigatórios responsáveis pelo processo de } \\
\text { desenvolvimento de software. }\end{array}$ & & \\
\hline Identificar processos da organização na ótica da norma. & $N$ & $S$ & $U$ \\
\hline Identificar os registros necessários e implementar os controles requeridos. & & \\
\hline Definir e alocar recursos necessários. & $N$ & $P$ & $C$ \\
\hline $\begin{array}{l}\text { Identificar os requisitos de competências necessários para as funções que afetam a qualidade dos } \\
\text { processos. }\end{array}$ & 4 & $S$ & $D$ \\
\hline Verificar necessidades de competências nos recursos humanos. & 6 & $S$ & $U$ \\
\hline Identificar treinamento necessário. & $S$ & $U$ \\
\hline Verificar eficácia do treinamento. & & & \\
\hline Verificar necessidades de recursos financeiros, infra-estrutura e meio ambiente. & 6 & $S$ & $U$ \\
\hline Implementação dos processos relacionados com o produto e com o desenvolvimento de software. & 6 & $S$ & $U$ \\
\hline Análise crítica pela alta administração. & 6 & $S$ & $U$ \\
\hline Validação e comprometimento da alta administração. & 6 & $S$ & $U$ \\
\hline Auditorias internas da qualidade. & $P$ & $C$ \\
\hline Processos de melhoria. & 5 & $S$ & $D$ \\
\hline Ações corretivas / preventivas. & 8 & $S$ & $D$ \\
\hline Ajustes finais. & 8 & $P$ & $C$ \\
\hline & 8 & $P$ & $R$ \\
\hline
\end{tabular}

As seguintes atividades foram também desenvolvidas na fábrica de software para a conclusão de seu processo de certificação ISO 9001:

- Seleção do órgão certificador.

- Pré-auditoria (presença do certificador e empresa).

- Auditoria de certificação (presença do certificador e empresa). 
Além dos procedimentos documentados citados anteriormente, um dos produtos gerados e validados frutos do processo de certificação foi a elaboração de uma metodologia de desenvolvmento de software para a fábrica de software.

\subsection{Produtos gerados e validados}

A Metodologia Unificada de Desenvolvimento de Software da SoftExport (MUSSE) é uma metodologia baseada no RUP [1], e adaptada à fábrica de software. Essa metodologia foi estruturada em conformidade com os requisitos da ISO 9001, implantada durante o processo de certificação e validada como o procedimento de realização do produto, segundo a Figura 1.

Foram definidos os papéis dos profissionais envolvidos e sua disposição organizacional na estrutura do projeto, e gerados diagramas de atividades, identificando todos os passos de cada fase do ciclo de vida, e atribuindo tarefas a todos os participantes, além da especificação dos artefatos resultantes das tarefas.

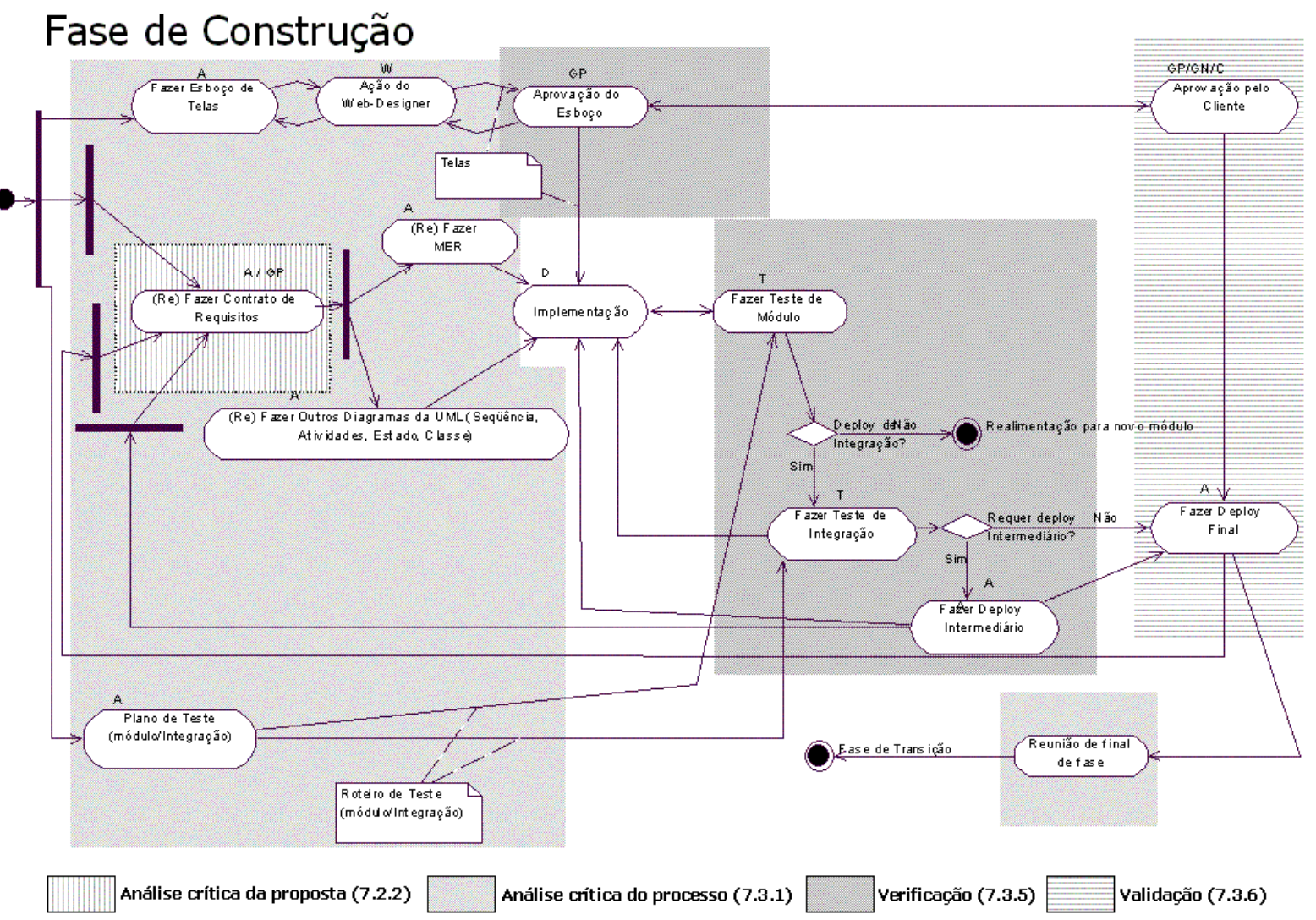

Figura 1. Fase de Construção do MUSSE indicando requisitos da ISO 9001

O processo de treinamento interno foi validado através do desenvolvimento de outro produto na fábrica de software, que foi denominado de CAFETEIRA. Esse produto estabelece as bases de conhecimentos necessários para todos as funções da estrutura organizacional e está incluído no requisito de qualificação profissional dos requisitos da ISO 9001. 
O produto CAFETEIRA está divido em 4 fases que abrangem as necessidades de qualificação para os colaboradores da fábrica de software em nível crescente de complexidade, conforme a Figura 2. Cada fase contempla atividades que estão programadas em seqüências e dispostas de forma mais otimizada de aproveitamento e dependências.

\begin{tabular}{|c|c|c|c|c|c|c|c|}
\hline PLANO GERAL & \multicolumn{3}{|c|}{ FASE 1} & FASE 2 & \multicolumn{2}{|c|}{ FASE 3} & FASE 4 \\
\hline 1 Ambiente de Desenvolvimento & Eclipse & cVs & BugCentral & & & & \\
\hline 2 Padrôes básicos de Programação & 00 & + & JAVA & & & & \\
\hline & & & & & & & \\
\hline 3 Padrões de Controle de Dados & & SQL & & JDBC & & & \\
\hline 4 Convençốes de Código de Programaçấo & & & CODE & I CONYFNTIONS & & & \\
\hline & & & CovL & COIAVLIVIOTOS & & & \\
\hline 5 Padrōes Avançados de Programaçã̃o & & & & JavaScript & Java(JSP) & & \\
\hline & & & & & & & \\
\hline 6 Padrỗes de Modelagem de Aplicaçốes & & & & UML-Básico & UML-Avançad & & \\
\hline 7 Padrốes de Controle de Dados genéricos & & & & XML-Básico & & & XML Avançado \\
\hline & & & & & & & \\
\hline 8 Estrutura-SE de Desenvolvimento & & & & FW-Básico & FW-Int & mediário & FW-Avançado \\
\hline 9 Arquitetura de Aplicaçōes & & & & & Arquitetura & e Aplicações & \\
\hline & & & & & & & \\
\hline 10 Aplicaçã̃o Piloto & & & & & Aplican & Piloto & \\
\hline 11 Processo de Desenvolvimento SE & & & SSE & MUSSE & & SSE & MUSSE \\
\hline & & & & & & & \\
\hline 12 Processo de Controle de Testes & & & & Junit-Básico & Junit-Int & imediário & Junit-Avançado \\
\hline 13 Padrốes de Projetos & & & & & & & 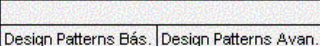 \\
\hline SGQ & & FASE 1 & & FASE 2 & & E 3 & FASE 4 \\
\hline & & & & & & & \\
\hline 1 Procedimentos Organizacionais & SQ.PO27 & & SQ.P021 & SQ.PO24 & & & \\
\hline 2 Procedimentos Fundamentais & & SQ.PF20 & & & & & \\
\hline 3 Procedimentos de Apoio & SQ.PA28 & SQ.PAD1 & SQ.PAD2 & SQ.PA05 & SQ.PA03 & & \\
\hline & & & & & & & \\
\hline 4 Produtos SE & & & & Abordagem Funcional & Abordagen & Operacional & Abordagem Arquitetural \\
\hline
\end{tabular}

Figura 2. Etapas e disciplinas necessárias para o desenvolvedor SE.

\section{As Lições Aprendidas}

Podemos citas como principais lições aprendidas ao longo do processo de certificação ISO 9001:2000 [3]:

- O apoio explícito da alta administração da empresa foi de fundamental relevância, uma vez que abriu aos participantes do processo de certificação todas as estruturas da empresa e de seus colaboradores, de forma prioritária;

- As definições de Visão, Política e objetivos da Qualidade motivaram a diretoria a definir e acompanhar indicadores, que realmente interessam e motivam o controle da estrutura organizacional;

- O levantamento e o estudo dos processos de desenvolvimento de software adotados pela empresa proporcionaram aos participantes do processo, uma rápida integração com as rotinas e com os desenvolvedores;

- O levantamento e o estudo de produtos de software da empresa identificou que vários desses produtos eram utilizados internamente nos processos de gerência e desenvolvimento. Alguns desses produtos já se adequavam a itens de controle obrigatório da norma;

- O estudo da norma ISO 9000:2000 [2] situou o grupo no ambiente de Sistema da Qualidade. O enfoque desenvolvido foi a identificação e a adequação de rotinas e produtos da empresa à norma.

- O mapeamento entre a ISO 9001:2000 [3] e a ISO/IEC 12207 [4] integrou os requisitos de certificação com os processos de ciclo de vida, possibilitando a divisão em três 
frentes de ação (processos organizacionais, fundamentais e de apoio) e facilitando o diálogo com todos os colaboradores da empresa;

- O seqüenciamento das atividades utilizado no processo de certificação ISO 9001:2000

[3] para a fábrica de software, que foi considerado satisfatório, é apresentado a seguir:

- Sistema de Gestão da Qualidade (seção 4 da norma): o estabelecimento, a documentação e a implementação de um sistema de qualidade, descrevendo a necessidade dos processos serem conhecidos e documentados.

- Gestão de Recursos (seção 6): apresenta os recursos disponíveis e necessários à prática da qualidade.

- Realização do Produto (seção 7): mostra a implementação do produto à luz dos procedimentos e requisitos do cliente.

- Responsabilidade da Direção (seção 5): integra a Alta Administração no próprio processo de implantação do $S G Q$, validando e adotando, perante todos os colaboradores, o processo da Qualidade.

- Medição, Análise e Melhoria (seção 8): descreve o processo de medição, análise e melhoria, podendo retornar a qualquer das etapas de implantação.

Desta maneira, o projeto de implantação do SGQ baseado na ISO/IEC 12207 [4] pode ser visto como um processo que segue a ISO 9001 [3] e usa suas atividades de medição, análise e melhoria (seção 8), para ajustar as outras atividades. A atividade de Responsabilidade da Direção assegura a validade e o alcance das propostas e reafirma o apoio necessário a todo o processo.

\section{Conclusão}

Este trabalho apresenta a experiência do processo de certificação da norma de qualidade ISO 9001:2000 [3] da fábrica de software SoftExport. O SGQ implantado a partir da ISO/IEC 12207 [4] tem assegurado que seu processo de desenvolvimento de produtos tenha um nível de controle, disciplina e repetibilidade, garantindo a qualidade de seus produtos de software gerados.

O processo de certificação foi conduzido através de um conjunto de atividades estruturadas a partir da ISO/IEC 12207, diferenciando-se da seqüência lógica de implantação dos itens da ISO 9001, levando a uma maior agilidade no processo de implantação do SGQ na fábrica de software.

\section{Referências}

[1] Booch, G.; Jacobson, I.; Rumbaugh, J., 1999, The Unified Software Development Process, Addison-Wesley.

[2] NBR ISO 9000, 2000a, Sistemas de gestão da qualidade - Fundamentos e vocabulário.

[3] NBR ISO 9001, 2000b, Sistemas de gestão da qualidade - Requisitos.

[4] NBR ISO/IEC 12207, 1998, Tecnologia da Informação: processos de ciclo de vida de software, ABNT, Rio de Janeiro.

[5] Rocha, A. R. C., Maldonado, J. C. e Weber, K. C., 2001, Qualidade de Software: Teoria e Prática, São Paulo, Prentice Hall.

[6] Weber. K. C., Rocha,A. R. C. e De Luca, J. C. M, 1999, Qualidade e Produtividade em Software, 2a . Edição, São Paulo, Makron Books. 